\title{
Nonlabeling and Nonexternal Indicator DNA Sensing Based on Ferrocene-terminated Probes Immobilized on Gold Film Electrode Arrays with Plasma and Acid Treatments
}

\author{
Hiroshi Aoki, ${ }^{*}$ Takeshi Sukegawa, ${ }^{\dagger}$ Masaki Torimura, and Tetsuya Nakazato \\ Environmental Management Research Institute, \\ National Institute of Advanced Industrial Science and Technology (AIST), \\ 16-1 Onogawa, Tsukuba, Ibaraki 305-8569, Japan
}

(Received September 30, 2019; accepted January 27, 2020)

Keywords: oligonucleotides, DNAs, sensor array chips, long-term storage, activation

We fabricated a novel sensor array chip that enables simple, rapid, and sequence-specific DNA detection without any process to label DNAs or any external electrochemical indicators. We found the conditions for the cleaning of electrode surfaces and the preparation of DNA sensor array chips. A long-term storage for several weeks inactivated photolithographically fabricated 384-ch microelectrode array chips before surface modification. We revealed that the treatments with $\mathrm{O}_{2}$ plasma and acidic solutions reactivated the long-term stored chips, and demonstrated that the DNA sensor arrays prepared from the reactivated chips detected DNAs sequence-specifically, similarly to freshly fabricated sensors. We found that feasible DNA sensor array chips can be prepared from the electrode array chips stored even for a long period, as long as the stored chips are treated before use with $\mathrm{O}_{2}$ plasma and acid solutions under optimized conditions.

\section{Introduction}

Oligonucleotides, especially RNAs, reflect biological information in living things. ${ }^{(1-3)}$ Many researchers have been interested in their attractive characteristics as biomarkers for the diagnoses of environmental chemical toxicity and biological diseases. ${ }^{(4-6)}$ Recently, the oligonucleotides used for environmental and biomedical testing have been extensively investigated as fast screening tools. ${ }^{(7-10)}$ Aiming at providing fast screening tools, many research efforts over the past few decades have been devoted to the development of numerous oligonucleotide detection techniques. They include optical, gravimetric, and electrochemical techniques. ${ }^{(11)}$ Electrochemical techniques have several notable merits compared with other techniques, such as a simple design and the use of low-power instruments, which contribute to size reduction and cost-effective oligonucleotide detection systems. ${ }^{(12)}$ Targeting simple and rapid screening techniques, many researchers have studied oligonucleotide detection methods based on electrochemical techniques.

\footnotetext{
*Corresponding author: e-mail: aoki-h@aist.go.jp

†Present address: Research Institute for Physical Measurement, National Institute of Advanced Industrial Science and Technology (AIST), 1-1-1 Umezono, Tsukuba, Ibaraki 305-8563, Japan 
For the development of simple and rapid screening techniques that are feasible in the environmental and biomedical fields as fast screening tools, we have studied electrochemical oligonucleotide detection techniques based on hybridization-amenable electrochemical signalswitching architectures without any labeling of target oligonucleotides. ${ }^{(13,14)}$ To improve the throughput of oligonucleotide detection, we have developed the 9 -ch, ${ }^{(15)} 96-\mathrm{ch}^{(16)}$ and 384-channel ${ }^{(17)}$ electrochemical array chips. The sensor array chips are photolithographically fabricated $\mathrm{Au} / \mathrm{Cr}$ - or $\mathrm{Au} / \mathrm{Ti}$-based electrodes modified with artificially synthesized oligonucleotide probes. The sensor areas are restricted to a diameter of around $300 \mu \mathrm{m}$ with photoresists. The chips realized the simultaneous detection of multiple environmentally and biomedically relevant RNA biomarker target sequences on a single chip. The chips showed sequence-specific responses upon the hybridization of the probes with the target sequences complementary to the probes in contrast to mismatch sequences.

The utilization of the chips as simple, rapid, and fast screening tools, basically based on the disposable use of the chips to prevent positive false results, requires reductions in the fabrication cost and unevenness of the chips. To control the quality to meet these goals, we fabricated the electrode array chips in large quantities and stored them for long periods until use. However, the long-term storage of the chips can lead to the adhesion of contaminants to the chip surfaces and the degradation of the photoresists on the chips. These are the problems that cause the inactivation of the electrode surfaces and make it difficult to prepare workable sensor array chips. To prepare the sensor array chips that are feasible in the environmental and biomedical fields, it is important to address these problems caused by the long-term storage and to demonstrate the sequence-specific detection of oligonucleotides by using the prepared sensor array chips.

To solve these problems, in this study, we fabricated a novel sensor array chip that enables simple, rapid, and sequence-specific DNA detection without any process to label DNAs (nonlabeling) or any external electrochemical indicators (nonindicator). We found the conditions for the cleaning of electrode surfaces and the concomitant preparation of oligonucleotide sensor array chips. First, we fabricated 384-ch microelectrode array chips photolithographically, stored them in a vacuum desiccator for several weeks or months, and, right before use, we activated the electrodes with the plasma treatment as a dry process and/or the chemical treatment as a wet process. The electrode surfaces were electrochemically evaluated on the basis of peak potential separations $\left(\Delta E_{\mathrm{p}}\right)$ of cyclic voltammograms $(\mathrm{CVs})$ of $\left[\mathrm{Fe}(\mathrm{CN})_{6}\right]^{4-}$ as an electroactive marker obtained for the surfaces. Then, we determined whether the treated electrodes on the chip can be used in feasible oligonucleotide sensors.

Here, we adopted a nonlabeling and nonindicator DNA detection technique reported in our previous paper with the aim of simple and rapid detection. ${ }^{(18)}$ The technique is based on a probe composed of a peptide nucleic acid (PNA) conjugated with a terminal ferrocene (Fc) (Fc-PNA). The architecture is based on the hybridization-triggered switching of the Fc redox reaction, which is controlled by increasing the rigidity from a single-stranded form to a double-stranded form, followed by the inaccessibility of the terminal Fc to the electrode surface.

As a result, the combination of the $\mathrm{O}_{2}$ plasma treatment for 2 min and the chemical treatment in $1 \%$ trifluoroacetic acid (TFA) for $3 \mathrm{~h}$ mostly activated the electrode surfaces. We found that 
the obtained CVs show the quasi-reversible redox reaction of $\left[\mathrm{Fe}(\mathrm{CN})_{6}\right]^{4-}$ on the electrodes and that $\Delta E_{\mathrm{p}}$ decreased to $180 \mathrm{mV}$ after the treatment. The treatment did not completely activate the electrode surfaces to show the reversible redox reaction. However, it was revealed that the sensors concomitantly prepared by immobilizing Fc-PNA on the electrodes showed sequencespecific responses to the target, similarly to those purely prepared as reported in our previous study. ${ }^{(18)}$ Moreover, the Fc-PNA density of $5.5 \times 10^{-10} \mathrm{~mol} / \mathrm{cm}^{2}$ on the electrode (gold film electrode surface) was 10 -fold higher than that on the gold disk electrode surface reported in our previous study. ${ }^{(18)}$ These results indicate that the sensors can be prepared to detect properly the target DNA, even if the electrode treatment did not activate the electrodes to show the reversible redox reaction. We concluded that the plasma and chemical treatments and Fc-PNA immobilization play an important role in cleaning the surface and in preparing workable DNA sensor array chips.

\section{Materials and Methods}

\subsection{Reagents}

The artificially synthesized probe was purchased from CBC (Tokyo, Japan), which was designed as a conjugate of PNA and other moieties, with the structure of Fc-O-GCA ACC TTC CCT ATT ACT CCA C-O-Cys- $\mathrm{NH}_{2}$ (Fc-PNA) with the sequence from the estrogen-responsive gene EGR3 [the accession number of which is X63741, from the National Center for Biotechnology Information (NCBI) ${ }^{(19)}$ ], where $\mathrm{O}$ and Cys denote an ethylene glycol linker and a cysteine moiety, respectively. The structure of the probe Fc-PNA is depicted in Fig. 1(a). DNAs were from Eurofins Genomics Japan (Tokyo, Japan), the sequences of which were 5' GTG GAG TAA TAG GGA AGG TTG C 3' (target DNA), complementary to the sequence of the probe, and 5' CGT GAA AGA CAG AAT 3' (mismatch DNA). The target and mismatch DNAs were used in this study as models of sample RNAs to evaluate the prepared sensor array chips. 6-Hydroxy-1-hexanethiol (HHT) was from Dojindo (Kumamoto, Japan). 10X phosphate-buffered saline (10X PBS), TFA, and hydrochloric acid $(\mathrm{HCl})$ were from Wako Chemical (Tokyo, Japan). 1X PBS was prepared from 10X PBS by diluting 10 times. All aqueous solutions were prepared with deionized and charcoal-treated water (specified resistance $>18.2 \mathrm{M} \Omega$ ), obtained using a Milli-Q reagent-grade water system (Millipore; Bedford, MA).

\subsection{Fabrication of electrode array chips}

A 384-ch microelectrode array chip (384 channels; with an electrode diameter of $300 \mu \mathrm{m}$ and an electrode interval of $1 \mathrm{~mm}$, Fig. 2) was fabricated in the manner previously reported ${ }^{(16,17,20)}$ or fabricated by NTT Advanced Technologies (Kanagawa, Japan) in the same manner as ours. Briefly, a glass substrate [size, $36 \times 36 \mathrm{~mm}^{2}$; thickness, $0.7 \mathrm{~mm}$, from Corning (\#1737, Corning; NY)] was sputtered with titanium (1 nm thickness) and gold (100 nm thickness) thin films using a CFS-4EP-LL sputtering apparatus (Shibaura Mechatronics; Kanagawa, Japan) to form Au/Ti 


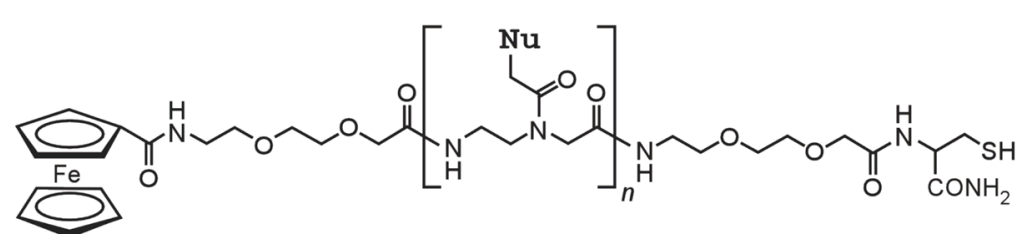

(a)

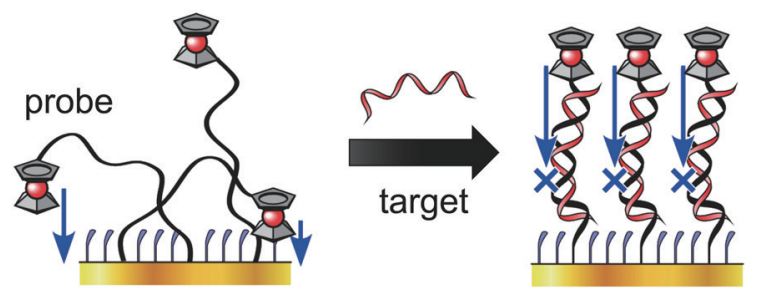

(b)

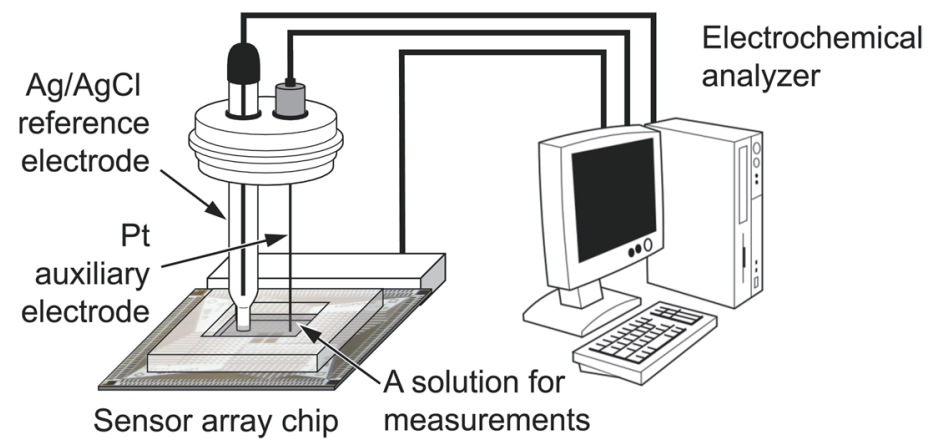

(c)

Fig. 1. (Color online) (a) Chemical structure of probe PNA. (b) Oligonucleotide detection based on switching controlled by hybridization-amenable flexibility change in the probe structure. (c) Experimental setup used in this study.

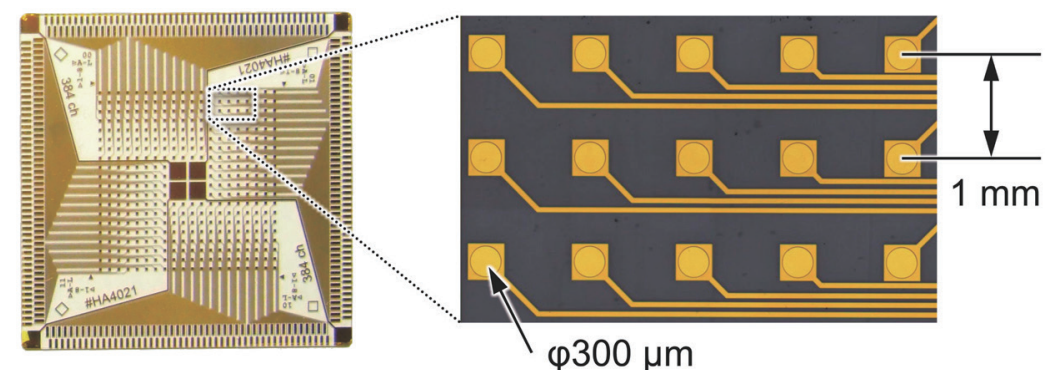

(a)

(b)

Fig. 2. (Color online) (a) Photolithographically fabricated 384-ch microelectrode array chip and (b) enlarged image of part of the chip. The diameter of each electrode is $300 \mu \mathrm{m}$ and the interval of the electrodes is $1 \mathrm{~mm}$.

films on the substrate. The Au/Ti-sputtered glass substrate was subjected to photolithography to pattern an electrode array. The chip has individual electrical terminals for each microelectrode to connect to an ALS 760C electrochemical analyzer [Bioanalytical Systems (BAS); Tokyo, Japan]. The chip was coated with an SU-8 3000 positive photoresist (Kayaku Microchem; Tokyo, Japan) as an electric insulation except for the surfaces of microelectrodes and electric terminals. Until use, the fabricated chips were stored in a vacuum desiccator for several weeks or months. 
The chip was cleaned prior to use with the combination of plasma and acid treatments. The $\mathrm{O}_{2}$ plasma was radiated on the chip with different radiation periods and powers using a PR301 plasma reactor (Yamato Scientific; Tokyo, Japan). The chip was cleaned with aqueous solutions containing $1 \%$ TFA or $1 \% \mathrm{HCl}$. The treated chips were used within a waiting period of $10 \mathrm{~min}$.

\subsection{Preparation of sensor array chips}

The probe Fc-PNA was immobilized on the electrode surfaces of the chips by dropping the aqueous solution of $100 \mu \mathrm{M}$ Fc-PNA in 1X PBS onto the electrode surfaces and storing the chips at room temperature for $24 \mathrm{~h}$. After washing with 1X PBS, the aqueous solution of $1 \mathrm{mM}$ HHT was dropped onto the electrode surfaces, and the chips were stored at room temperature for more than $4 \mathrm{~h}$, followed by washing the surfaces with 1X PBS. The aqueous solutions of $100 \mu \mathrm{M}$ target and mismatch DNAs in 1X PBS were dropped onto the prepared sensor arrays, and the chips were stored at room temperature for $24 \mathrm{~h}$. The electrochemical measurement was performed after washing the sensor array chip with 1X PBS. The working principle of the sensor responding to DNAs is shown in Fig. 1(b), depicting the DNA detection system with nonlabeling signal transduction. The hybridization of a probe PNA with the target DNA complementary to the probe makes the probe structure more rigid owing to the double-helix formation, inhibiting the access of the terminal $\mathrm{Fc}$ moiety to the electrode surface and electron transfer from the $\mathrm{Fc}$ to the surface. This induces the decrease in the observed current for the redox reaction of the Fc. As a result, the hybridization-amenable flexibility change in the probe structure shows the presence of the target DNA based on electrochemical signal switching.

\subsection{Electrochemical detection of DNAs}

The electrochemical measurement was conducted using a three-electrode configuration of the prepared electrode on the chip as the working electrode, a $\mathrm{Ag} / \mathrm{AgCl}$ reference electrode (internal solution: $3 \mathrm{M} \mathrm{NaCl}$ ), and a Pt auxiliary electrode. It was also conducted using an ALS $760 \mathrm{C}$ electrochemical analyzer and ALS 760C analysis software (BAS). The electrochemical cell was constructed similarly to that in the previous report. ${ }^{(17)}$ Briefly, the prepared chip was connected to ALS $760 \mathrm{C}$ with the contact pads formed on one edge of the chip using a chip adaptor with contact probes (Yokowo; Tokyo, Japan). The edge has 96 contact pads and each pad is connected to one sensor on the chip. A silicone rubber frame (size, $28 \times 28 \mathrm{~mm}^{2}$; thickness, $3 \mathrm{~mm}$; frame width, $3 \mathrm{~mm}$ ) was placed on the prepared chip filled with $1 \mathrm{~mL}$ of the measurement solution, with the reference and auxiliary electrodes inserted into the solution perpendicularly and close to the chip [Fig. 1(c)].

An electrochemical investigation on the dependence of the electrode array chips on the cleaning treatments was conducted in $1 \mathrm{X}$ PBS containing $1 \mathrm{mM} \mathrm{K}_{4}\left[\mathrm{Fe}(\mathrm{CN})_{6}\right]$ as an electroactive marker at room temperature. The electrochemical activity of the electrode surface was evaluated by cyclic voltammetry on the basis of $\Delta E_{\mathrm{p}}$ and the difference between the oxidative and reductive peak current potentials in the recorded CVs. For cyclic voltammetry, the potential was scanned from 0 to $+0.5 \mathrm{~V}$ and again back to $0 \mathrm{~V}$ at a rate of $0.1 \mathrm{~V} \mathrm{~s}^{-1}$. The electrochemical 
measurement of sensor responses for the sensor array chips before and after the oligonucleotides was conducted in 1X PBS at room temperature. The sensor response was evaluated by square wave voltammetry on the basis of the peak current decrease in the recorded square wave voltammograms (SWVs). For square wave voltammetry, the potential was scanned from 0 to $+0.65 \mathrm{~V}$ at a potential step of $2 \mathrm{mV}$, a potential amplitude of $25 \mathrm{mV}$, and a frequency of $50 \mathrm{~Hz}$. The sensor responses to the DNAs were expressed as the normalized current change of $\left(i_{0}-i\right) / i_{0}$ in the measured SWVs, the ratio of the peak current decrease $\left(i_{0}-i\right)$ after incubation in the solution containing DNAs to the peak current $\left(i_{0}\right)$ before incubation, similarly to our previous reports. ${ }^{(15-17)}$ The normalized current change was depicted on a gray scale ranging from white (low) to black (high). Each SWV was subjected to a baseline fitting algorithm in the ALS 760C analysis software to subtract a baseline from the SWV before normalization.

\section{Results and Discussion}

\subsection{Characterization of the electrode array chips and their activation with plasma treatment}

Electrodes on the fabricated electrode array chips stored in a vacuum desiccator were electrochemically investigated on the basis of the $\Delta E_{\mathrm{p}}$ values for CVs of an electroactive marker $\left[\mathrm{Fe}(\mathrm{CN})_{6}\right]^{4-}$. The investigation showed large $\Delta E_{\mathrm{p}}$ values with large variations (sometimes more than $500 \mathrm{mV}$ ), indicating a slow electron transfer of the redox reaction of $\left[\mathrm{Fe}(\mathrm{CN})_{6}\right]^{4-}$ at electrode surfaces. We considered that the electrode surfaces might be inactive because of the adsorption of some impurities derived from the photoresist coating on the chips or organic compounds from the desiccator walls during the storage in the desiccator. The $\mathrm{O}_{2}$ plasma treatment of the chip surfaces then decreased the $\Delta E_{\mathrm{p}}$ values, showing the activation of the electrode surfaces. It was also revealed that long plasma treatment periods and large plasma powers, on the other hand, worsen the electrode surfaces with $\Delta E_{\mathrm{p}}$ values larger than those even before treatment. In a detailed investigation, a 2 min plasma treatment with a plasma power of $100 \mathrm{~W}$, and a $\mathrm{O}_{2}$ flow rate of $30 \mathrm{~mL} / \mathrm{min}$ showed the smallest $\Delta E_{\mathrm{p}}$ values [Figs. 3(a) and 3(b)]. Generally, it is known that plasma-treated gold surfaces form a gold oxide film on the surface, and that, resultantly, the surface becomes inactivated. ${ }^{(21,22)}$ Moreover, the treatment of the formed oxide film with acidic solutions can reactivate and change the film back again to the original active gold film. ${ }^{(21,22)}$ Therefore, we examined the treatment with acidic solutions, including $1 \%$ TFA and $1 \% \mathrm{HCl}$ aqueous solutions, as well as the plasma treatment. Figure 3(a) shows the dependence of $\Delta E_{\mathrm{p}}$ values in the measured $\mathrm{CVs}$ for $\left[\mathrm{Fe}(\mathrm{CN})_{6}\right]^{4-}$ on both the periods of plasma treatment and soaking in 1\% TFA. The soaking period was from 1 to $4 \mathrm{~h}$. $\Delta E_{\mathrm{p}}$ was observed to be minimum in the case of soaking for $3 \mathrm{~h}$, indicating the reactivation of the gold electrode surfaces.

In the case of soaking for more than $4 \mathrm{~h}$, however, $\Delta E_{\mathrm{p}}$ increased, showing that the longterm soaking even deactivates the gold electrode surfaces. Moreover, in the case of $1 \% \mathrm{HCl}$, soaking the electrode for $0.5 \mathrm{~h}$ increased $\Delta E_{\mathrm{p}}$ and deactivated the electrode surfaces. It was also observed that the photoresist of the surfaces peeled off from the chip after the long-term 


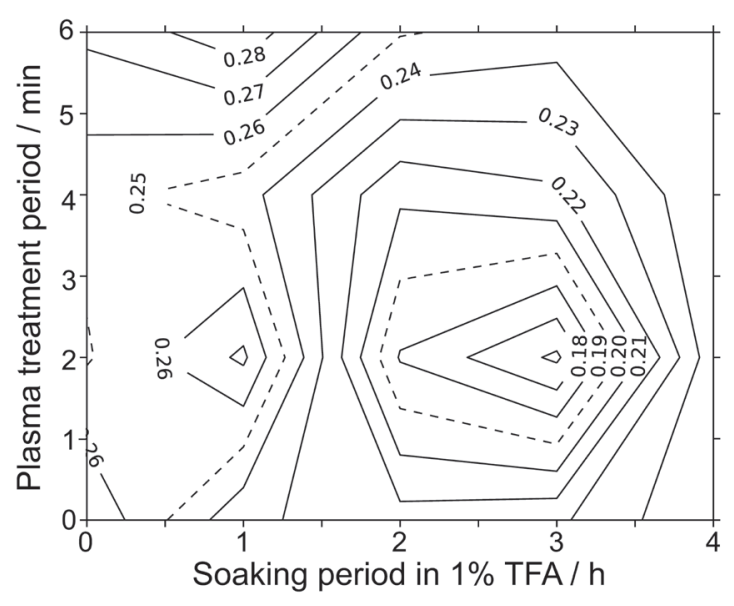

(a)

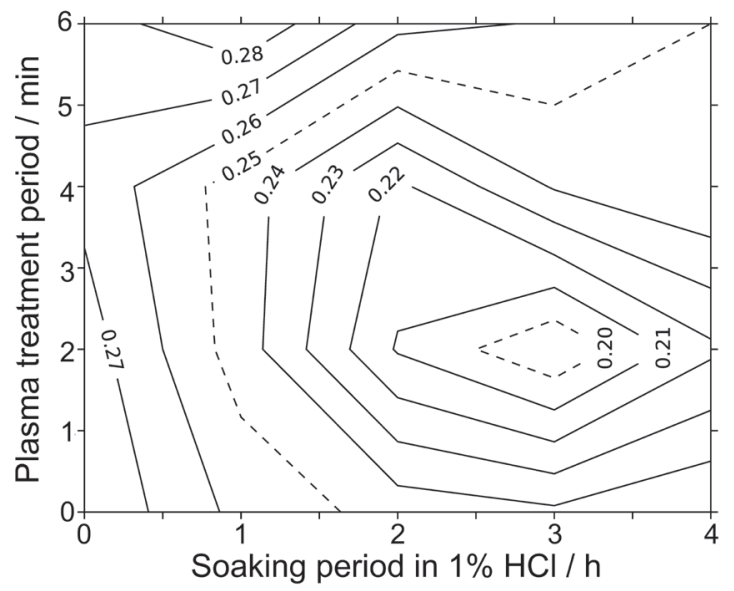

(b)

Fig. 3. Dependence of $\Delta E_{\mathrm{p}}$ for $\mathrm{CVs}$ measured in $1 \mathrm{X}$ PBS containing $1 \mathrm{mM} \mathrm{K} \mathrm{K}_{4}\left[\mathrm{Fe}(\mathrm{CN})_{6}\right]$ on plasma treatment and soaking periods in $1 \% \mathrm{TFA}$ (a) or $1 \% \mathrm{HCl}$ (b) aqueous solution.

soaking in acidic solutions. The reason for these phenomena was not further studied, but the possible reason might be the deterioration of the chip surfaces. In the next study, we activated the gold electrode surfaces by $\mathrm{O}_{2}$ plasma treatment for 2 min and soaking treatment in $1 \%$ TFA for $3 \mathrm{~h}$.

\subsection{Electrochemical nonlabeling detection of oligonucleotides}

The artificially synthesized probe Fc-PNA was immobilized on the activated gold electrode surface. In the previous study based on gold disk electrodes, ${ }^{(18)}$ electrochemical demonstrations using CVs revealed that Fc-PNA makes a binary monolayer with short-chained thiols on the gold surface and that the surface density of Fc-PNA was $3.3 \times 10^{-11} \mathrm{~mol} / \mathrm{cm}^{2}$ (considering the surface roughness factor of 1.3, calculated from the oxygen adsorption/desorption peaks measured in $1 \mathrm{M} \mathrm{H}_{2} \mathrm{SO}_{4}$ ), where a molecule stably has $\mathrm{Fc}$ on the surface. In this study, the surface density of Fc-PNA on the gold-sputtered film electrodes on the chip was calculated to be $5.5 \times 10^{-10} \mathrm{~mol} / \mathrm{cm}^{2}$ without considering the surface roughness. The observation of the gold electrode surface showed that the gold film electrode has a rougher surface than the gold disk electrode, indicating that the former might have a 10 -fold larger surface roughness factor than the latter. This large roughness gives a positive contribution to help increase the intensity of electrochemical current signals by increasing the real electrode surface areas. In this study, however, we could not demonstrate the roughness factor of the gold film electrodes because the film electrodes were dissolved during the electrochemistry in $1 \mathrm{M} \mathrm{H}_{2} \mathrm{SO}_{4}$ to induce the factor.

Figure 4 shows SWVs obtained before and after the hybridization of the target DNA with Fc-PNA on the sensor electrode. Before hybridization, a large oxidation peak for the Fc of FcPNA was observed with the oxidation current of $35.5 \mathrm{nA}$ (solid line), on the one hand. On the other hand, after the hybridization of the target by incubating the sensor chip in the solution containing the target DNA $(100 \mu \mathrm{M})$ complementary to the probe Fc-PNA, the oxidation peak 


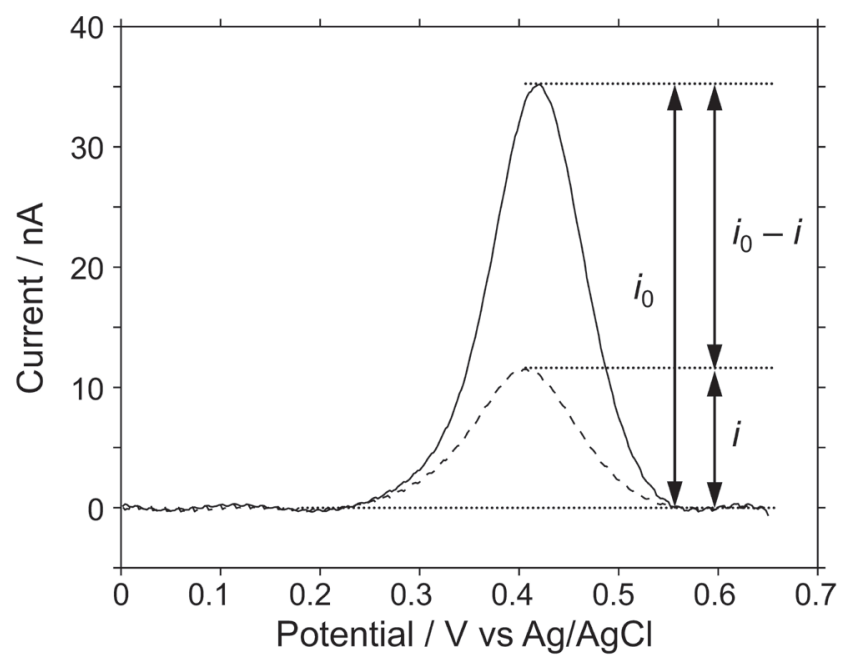

Fig. 4. Typical SWVs measured in PBS with (solid) and without (dashed) $100 \mu \mathrm{M}$ target oligonucleotide. The sensor response to the target oligonucleotide was expressed as the ratio of the current decrease $\left(i_{0}-i\right)$ to the peak current $\left(i_{0}\right)$ for the measured SWVs, $\left(i_{0}-i\right) / i_{0}$.

for Fc was observed with the oxidation current of $11.8 \mathrm{nA}$ (dashed line). The peak showed a marked decrease of $66.9 \%$ after hybridization. This peak decrease was attributed to the increase in the rigidity of the probe structure caused by duplex formation, inhibiting the access of the terminal Fc moiety to the electrode surface. In the case of the mismatch DNA, however, the sensor did not show any decrease in peak current. This indicates the sequencespecific detection of the target DNA for the prepared sensors. Consequently, we demonstrated the ability of the sensor array chip to detect DNAs sequence-specifically without any process to label DNAs or any external electrochemical indicators, contributing to simple and rapid DNA detection. In the next study, the sensor responses to the DNAs were expressed as the normalized current change of $\left(i_{0}-i\right) / i_{0}$ in the measured SWVs (shown in Fig. 4).

Fc-PNA was immobilized on multiple microelectrodes arrayed on a single chip to prepare the sensor array chip. Figure 5 shows the sensor responses for adjoining 10 prepared sensors before and after incubation in the solution containing $100 \mu \mathrm{M}$ target DNA. The average response was calculated to be $0.672 \pm 0.0497(n=10)$, showing high reproducibility. This result is similar to the previous result for the gold disk electrode, $0.724 \pm 0.0711(n=3)$, indicating that we developed a sensor array chip with a DNA detection ability similar to that of the gold disk electrode. ${ }^{(18)}$

\subsection{Activation of gold film electrodes after long-term storage}

In the previous study using 384-ch electrode array chips, the $\Delta E_{\mathrm{p}}$ values in CVs of $1 \mathrm{mM}$ $\left[\mathrm{Fe}(\mathrm{CN})_{6}\right]^{4-}$ marker were used for the evaluation of the activation of plasma treatment. ${ }^{(17)}$ The $\Delta E_{\mathrm{p}}$ values were changed after the plasma treatment from 220 to $80 \mathrm{mV}$ or from 265 to $100 \mathrm{mV}$ for electrodes in the central or peripheral area on a single chip. In this study, however, the 

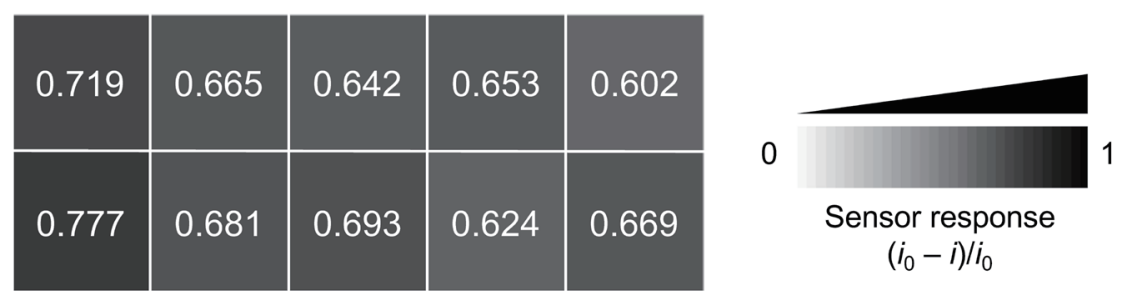

Fig. 5. Typical sensor responses to the target oligonucleotide $(n=10)$. The normalized current change of $\left(i_{0}-i\right) / i_{0}$ was depicted on a gray scale ranging from white (low) to black (high).

$\Delta E_{\mathrm{p}}$ values were around $180 \mathrm{mV}$ at best even after the treatment. The electrode array chip was stored for several weeks or months after the fabrication. It is probable that longer-term storage has worsened the electrode surface to be inactivated.

In contrast, after the treatment, the chips were reactivated and used for the preparation of sensors. The sensor chip showed the sequence-specific DNA detection similarly to the previous sensors based on gold disk electrodes, whose $\Delta E_{\mathrm{p}}$ was about $60 \mathrm{mV}$ before Fc-PNA immobilization, indicating an ideal reversible redox reaction of the $\left[\mathrm{Fe}(\mathrm{CN})_{6}\right]^{4-}$ marker. ${ }^{(18)}$ The immobilization process may activate the electrode surface. The adsorbed impurities, which could not be removed by the plasma and acid treatments, may be washed away during soaking in solvents for hours or by replacement with Fc-PNA and HHT on the surface. ${ }^{(23)}$ This indicates that the replacement of contaminants with these molecules on the electrode surface during the immobilization is also important to clean the surface other than the above-mentioned plasma or chemical treatment process. This information might be helpful in the easy preparation of the chips in the large-scale production processes. The utilization of the chips is basically based on disposable use in terms of the avoidance of false positive results. Therefore, the reductions in the fabrication cost and unevenness of the chips are required. The fabrication of the chips in large quantities, followed by storage for long periods until use can meet these goals.

In this study, the electrode array chips were used within a waiting period of $10 \mathrm{~min}$ after the activation treatment. Although we did not determine the changes in electrode properties with longer waiting periods, a waiting period of at least $10 \mathrm{~min}$ is sufficiently long for preparing to move to the next surface modification process in time. This might also appeal to the feasibility of the thus studied activation treatment.

\section{Conclusions}

In this study, we found the conditions for the cleaning of electrode surfaces and the preparation of oligonucleotide sensor array chips that enable the simple and rapid detection of oligonucleotides without labeling the target or adding external electrochemical indicators, based on photolithographically fabricated 384-ch microelectrode array chips. After a longterm storage of the microelectrode array chips, we activated electrode surfaces by $\mathrm{O}_{2}$ plasma treatment for $2 \mathrm{~min}$ and soaking treatment in 1\% TFA for $3 \mathrm{~h}$. The surface density of Fc-PNA on the gold film electrodes on the chip was calculated to be $5.5 \times 10^{-10} \mathrm{~mol} / \mathrm{cm}^{2}$, indicating 
that the electrodes have a 10-fold larger surface roughness factor than the gold disk electrode. Consequently, the sensor array chip detected DNAs sequence-specifically without any process to label DNAs or any external electrochemical indicators, contributing to simple and rapid DNA detection.

We also concluded that the immobilization of Fc-PNA and HHT is also important to clean the surface by other than the plasma or chemical treatment process. This might be helpful in the easy preparation of the chips in large-scale production processes. This is because the utilization of the chips is basically based on disposable use to avoid false positive results, and therefore, the reductions in the fabrication cost and unevenness of the chips are required. The fabrication of the chips in large quantities, followed by storage for long periods until use can meet these goals. We believe that the knowledge obtained in this study helps in optimizing the treatment conditions of the electrode surfaces.

To the best our knowledge, dominant techniques need the labeling of targets, external indicators, or complicated detection systems for signal amplification, ${ }^{(14,24)}$ and in some cases, an elaborate nanotechnology. ${ }^{(10,25,26)}$ However, simple, rapid, and "one-time" disposable devices to avoid false positive results without any labeling of targets or any external indicators are required for reliable oligonucleotide detection in the environmental and biomedical fields. ${ }^{(17,27)}$ Our technique might contribute to developing practical oligonucleotide sensor array chips that can make environmental and biomedical diagnoses simpler and easier to perform.

\section{Acknowledgments}

This work was partially supported by Suzuken Memorial Foundation and JSPS KAKENHI Grant Number JP19K05536 (awarded to HA).

\section{References}

1 J. Borlak: Handbook of Toxicogenomics (Wiley-VCH, New York, 2005).

2 J. T. Mendell and E. N. Olson: Cell 148 (2012) 1172. https://doi.org/10.1016/j.cell.2012.02.005

3 J. Wang and Q. Cui: J. Nucleic Acids 2012 (2012) 978384. https://doi.org/10.1155/2012/978384

4 A. H. Harrill, S. D. McCullough, C. E. Wood, J. J. Kahle, and B. N. Chorley: Toxicol. Sci. 152 (2016) 264. https://doi.org/10.1093/toxsci/kfw090

5 S. Campion, J. Aubrecht, K. Boekelheide, D. W. Brewster, V. S. Vaidya, L. Anderson, D. Burt, E. Dere, K. Hwang, S. Pacheco, J. Saikumar, S. Schomaker, M. Sigman, and F. Goodsaid: Expert Opin. Drug Metab. Toxicol. 9 (2017) 1391. https://doi.org/10.1517/17425255.2013.827170

6 J. Hayes, P. P. Peruzzi, and S. Lawler: Trends Mol. Med. 20 (2014) 460. https://doi.org/10.1016/ j.molmed.2014.06.005

7 B. Alexander-Dann, L. L. Pruteanu, E. Oerton, N. Sharma, I. Berindan-Neagoe, D. Módos, and A. Bender: Mol. Omics 14 (2018) 218. https://doi.org/10.1039/c8mo00042e

8 Y. C. Huang: J. Toxicol. Environ. Health B 16 (2013) 381. https://doi.org/10.1080/10937404.2013.832649

9 R. M. Graybill and R. C. Bailey: Anal. Chem. 88 (2016) 431. https://doi.org/10.1021/acs.analchem.5b04679

10 R. Fiammengo: Biomark. Med. 11 (2017) 69. https://doi.org/10.2217/bmm-2016-0195

11 A. Sassolas, B. D. Leca-Bouvier, and L. J. Blum: Chem. Rev. 108 (2008) 109. https://doi.org/10.1021/cr0684467

12 E. Paleček and M. Bartošík: Chem. Rev. 112 (2012) 3427. https://doi.org/10.1021/cr200303p

13 H. Aoki: Bunseki Kagaku 61 (2012) 763.

14 H. Aoki: Chem. Asian J. 10 (2015) 2560. https://doi.org/10.1002/asia.201500449

15 H. Aoki, A. Kitajima, and H. Tao: Sens. Mater. 22 (2010) 327.

16 H. Aoki, A. Kitajima, and H. Tao: Anal. Sci. 26 (2010) 367. https://doi.org/10.2116/analsci.26.367 
17 H. Aoki, M. Torimura, and T. Nakazato: Biosens. Bioelectron. 136 (2019) 76. https://doi.org/10.1016/ j.bios.2019.04.047

18 H. Aoki and H. Tao: Analyst 132 (2007) 784. https://doi.org/10.1039/b704214k

19 National Center for Biotechnology Information. https://www.ncbi.nlm.nih.gov/ (accessed January 2020).

20 H. Aoki, A. Kitajima, and H. Tao: Supramol. Chem. 22 (2010) 455. https://doi.org/10.1080/10610278.2010.4860 34

21 T. Sakata, H. Ishii, N. Sato, K. Kuwahara, and K. Machida: Surface treatment method of gold. JP patent 4675702, 2011.

22 T. Sakata, H. Ishii, N. Sato, K. Kuwahara, and K. Machida: Surface treatment method of gold. JP patent 5379117, 2013.

23 H. O. Finklea: Electrochemistry of Organized Monolayers of Thiols and Related Molecules on Electrodes: Electroanalytical Chemistry, A. J. Bard and I. Rubinstein, Eds. (Marcel Dekker, New York, 1996) Vol. 19, p. 109.

24 F. Wang, X. Liu, and I. Willner: Angew. Chem. Int. Ed. 54 (2015) 1098. https://doi.org/10.1002/anie.201404652

25 Oxford Nanopore Technologies. https://nanoporetech.com/ (accessed January 2020).

26 Pac Bio.: https://www.pacb.com/ (accessed January 2020).

27 H. Aoki, R. M. Corn, and B. Matthews: Biosens. Bioelectron. 142 (2019) 111565. https://doi.org/10.1016/ j.bios.2019.111565

\section{About the Authors}

Hiroshi Aoki received his B.S., M.S., and Ph.D. degrees from The University of Tokyo, Japan, in 1996, 1998, and 2001, respectively. From 2001 to 2004, he was a postdoctoral fellow of the Japan Society for the Promotion of Science (PD) at The University of Tokyo and then a postdoctoral fellow at AIST. From 2004 to 2009, he worked as a research scientist at AIST. Since 2009, he has been a senior research scientist at AIST. His research interests are in the simple and rapid sensing for DNAs and RNAs and its applications to the evaluation of chemical toxicity in cellular and ecological systems. (aoki-h@aist.go.jp)

Takeshi Sukegawa received his B.S. degree from Ibaraki University, Japan in 1989. From 1989 to 2013, he worked at NEC Ibaraki (the name of which was changed to Solectron Ibaraki in 2001 and to Flextronics Ibaraki in 2005), Japan. Since 2014, he has been a technical scientist at AIST. His research interests are in micro-electromechanical systems (MEMS), bioengineering, and sensors. (mcz-sukegawa@aist.go.jp)

Masaki Torimura received his B.S. and M.S. degrees from Gifu Pharmaceutical University, Japan, in 1991 and 1993, respectively, and his Ph.D. degree from Kyoto University, Japan, in 1999. From 2004 to 2009, he worked as a research scientist at AIST. Since 2010 and 2018, he has been a group leader and a deputy director, respectively, in the Environmental Measurement Research Institute (EMRI) at AIST. His research interests are in bio-electrochemistry and separation sciences. (torimura-masaki@aist.go.jp)

Tetsuya Nakazato received his Ph.D. degree from Kyushu University in Japan in 1996. From 1996 to 1999, he was a postdoctoral fellow of the Japan Science and Technology Agency. From 1999 to 2001, he was a research scientist at the National Institute of Resource and Environment. 
From 2001 to 2017, he was a research scientist and then a senior research scientist at AIST. Since 2017, he has been a group leader in EMRI at AIST. His research interests are in the speciation analysis and on-site monitoring of elements in the environment and in vivo and also the relationship between the dynamics of elements and biological responses.

(tet.nakazato@aist.go.jp) 